\title{
Preisach model for the simulation of ferroelectric capacitors
}

\author{
Andrei T. Bartic, ${ }^{\text {a) }}$ Dirk J. Wouters, and Herman E. Maes \\ IMEC, Kapeldreef 75, B-3001 Leuven, Belgium \\ Jürgen T. Rickes and Rainer M. Waser \\ Research Center Jülich, D-52425 Jülich, Germany
}

(Received 18 February 2000; accepted for publication 2 November 2000)

\begin{abstract}
The emerging ferroelectric technology needs a reliable model for the simulation of the ferroelectric capacitors. This model would play a crucial role in designing new ferroelectric nonvolatile memories. As a main requirement, such a model must allow the calculation of the polarization variations for an arbitrary voltage applied to the ferroelectric. However, in spite of the large efforts made in modeling, most of the existing solutions fail to satisfy the above requirement or lack a minimal physical background. To address these problems, we developed a model based on a ferroelectric interpretation of the Preisach theory of hysteresis. In this articles, we try to elucidate how this theory, initially developed for ferromagnetic particles, can be adapted to the ferroelectric materials, despite the many differences between the two. Because the Preisach theory assumes a distribution of the coercitive voltages, we try to clarify its physical meaning in the case of the ferroelectric materials and propose a methodology to determine this distribution experimentally. To facilitate the implementation of the model, the experimental results are then fitted by an analytic function and the whole bidimensional distribution is calculated using a linear approximation. To evaluate the validity of the model, we performed simulations using the Spectre ${ }^{\circledR}$ circuit simulator and the results are in very good agreement with the measurements for the saturated hysteresis loops. The differences existing for the partial loops are mainly due to the linear approximation used for the Preisach distribution. This model can be successfully used for the design of the real memories. (C) 2001 American Institute of Physics. [DOI: 10.1063/1.1335639]
\end{abstract}

\section{INTRODUCTION}

A model that allows the calculation of the polarization variations or of the switching current for arbitrary voltage variations is needed for the design of the ferroelectric memories. The model should be easy to implement in a circuit simulator and to calibrate based on the experimental measurements. An expression for the switching current as a function of time was proposed by Fatuzzo and Merz ${ }^{1}$ by analyzing the domain wall kinetics. This work was continued by Ishibashi and Takagi ${ }^{2}$ who developed, based on Kolmogorov's theory of crystal growth, an easier method for calculating the switching current. However these models can be used only for the very particular case of a voltage step applied to the ferroelectric capacitor. ${ }^{3-5}$

A general expression of the switching current as a function of voltage was experimentally found by Drougard ${ }^{6}$ for ceramic samples and successfully used to simulate saturated and nonsaturated hysteresis loops. ${ }^{7,8}$ However it is very difficult for such an expression to be experimentally determined in thin films due to the very fast switching characterizing these systems.

Miller et al. ${ }^{9}$ assumed a tanh relation between the polarization and the voltage and they simulated the saturated and the nonsaturated hysteresis loops. But lack of a solid theory behind the equations causes the nonsaturated hysteresis loops

\footnotetext{
a) Author to whom correspondence should be addressed; electronic mail: Andrei.Bartic@imec.be
}

to be calculated by scaling the saturated loops with a rather arbitrary factor.

To address the previous problems, distribution models can be used. Such a model, using a one dimensional distribution of the domain wall coercitive voltages, was first proposed by Evans and Bullington. ${ }^{10}$ However this model employs a very simple memory mechanism, which memorizes only the last voltage turning point and therefore fails to describe the switching details for an arbitrary voltage. Although no limitation on the number of turning points is specified by the authors, the algorithm of adding and deleting these points is of the utmost importance as will be clearly shown in the following sections. Therefore, we developed a model based on the Preisach theory of hysteresis. ${ }^{11}$ The central point of this model is the assumption that the ferroelectric has a bidimensional distribution of coercitive fields, called the Preisach distribution, which clarifies the necessity of using a more complex memory mechanism using more than one voltage turning point. This approach was followed by Jiang et al. ${ }^{12}$ and Goebel et al. ${ }^{13}$ However, the implications of applying this theory to ferroelectric materials were not considered in previous articles. Furthermore, the distribution was not experimentally determined but rather chosen to obtain a good fit for the hysteresis loops.

This article discusses how the Preisach model can be adapted for ferroelectric materials. In contrast with previous work, ${ }^{12,13}$ we measure the distribution and then by applying the Preisach model the hysteresis loops are simulated using the Spectre ${ }^{\circledR}$ circuit simulator. ${ }^{14}$ Simulated hysteresis loops 


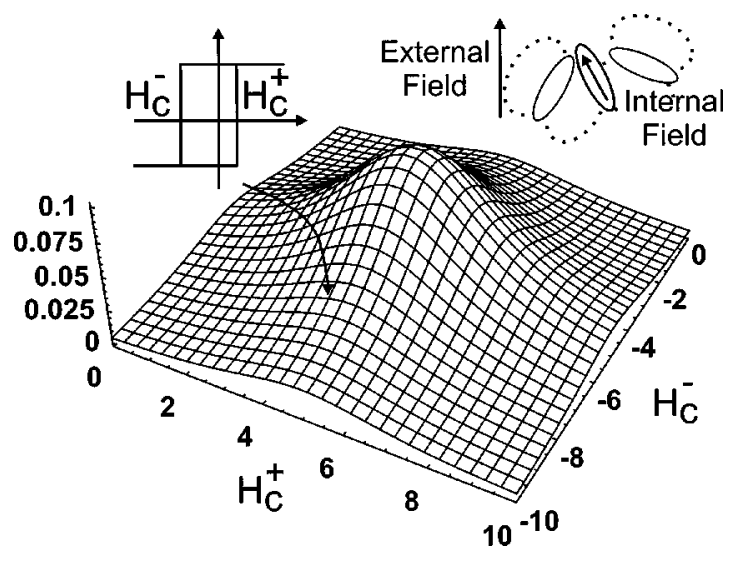

FIG. 1. In the case of magnetic particle ensemble, the interaction between magnetic particles (inset) is translated into an internal magnetic field. The internal field will shift the hysteresis loop such that the coercitive fields will not be equal anymore. A point of the Preisach distribution represents the number of particles with a certain pair of coercitive fields.

show good agreement with the experiment and therefore the model can be used for ferroelectric memory design.

\section{THE PREISACH THEORY}

In order to apply the Preisach theory to ferroelectric thin films it is important to clarify the physical meaning of the Preisach distribution and to analyze its values for the different regions of the Preisach plane. The Preisach model was initially proposed to describe the hysteresis of magnetic particle ensembles. These particles interact with each other and the interaction is modeled as an internal magnetic field that shifts their hysteresis loops, such that the coercitive fields will be different in absolute values (see inset of Fig. 1). The shift will depend on the particle local environment leading to a distribution of positive and negative coercitive fields. This so-called Preisach distribution gives the number of particles of the ensemble with a certain pair of coercitive fields (see Fig. 1).

In spite of its magnetic origin, the model can be regarded entirely from a mathematical point of view. ${ }^{11}$ In this way, it can be applied to other systems with hysteresis, such as ferroelectric thin films. However, it makes many assumptions that have to be verified for ferroelectric materials, and moreover restricting ourselves only to the mathematical treatment would set a strong limitation on its future developments.

The physical interpretation of the Preisach model relies mainly on finding the meaning of the Preisach distribution for ferroelectric thin films. At the microscopic level two interpretations have been proposed: (1) it could refer to a coercitive fields distribution of the ferroelectric dipoles ${ }^{13}$ or (2) it could refer to the threshold voltage at which the independent domains switch. ${ }^{10}$ The first theory is somewhat acceptable because the switching in ferroelectric materials does not take place dipole by dipole, but through domain walls movement. The second theory, in spite of the dramatic simplification of the switching process, is closer to the actual situation. The voltage at which the different domain wall moves is affected by the presence of defects, grain boundaries, or

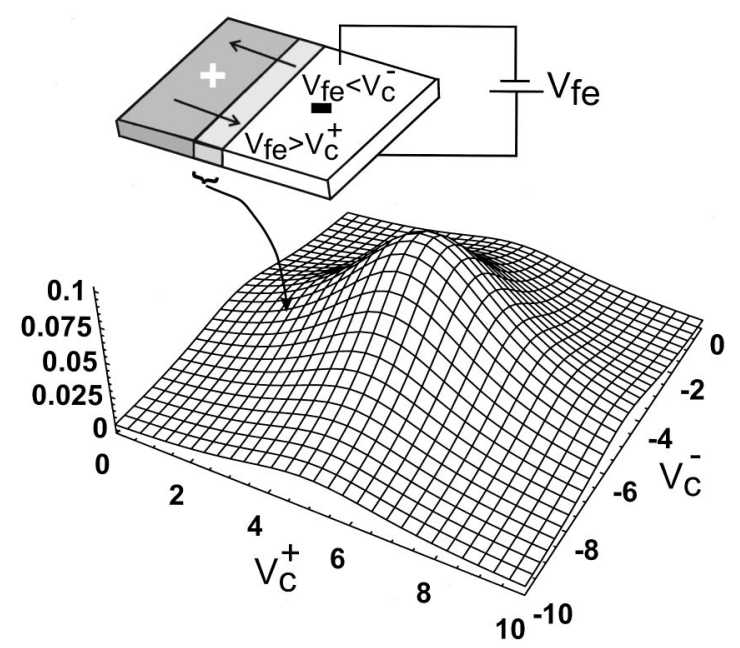

FIG. 2. For ferroelectric thin films the Preisach distribution represents the charge variation corresponding to a point, $\left(V_{c}^{+}, V_{c}^{-}\right)$in the plane. When $V_{\mathrm{fe}}>V_{c}^{+}$the region corresponding to positive oriented domains increases. Conversely, this region decreases by the same amount when $V_{\mathrm{fe}}<V_{c}^{-}$.

stress. ${ }^{10}$ The different local environments will lead to a distribution in the voltages at which the switching of the different domains in the ferroelectric takes place.

At the macroscopic level the interpretation of the distribution is very straightforward. The switching gives rise to polarization variation that can be measured experimentally. The Preisach distribution thus gives the polarization variation corresponding to a point $\left(V_{c}^{+}, V_{c}^{-}\right)$, in the Preisach plane (see Fig. 2). When the voltage over the ferroelectric capacitor $V_{\mathrm{fe}}$ equals $V_{c}^{+}$there is an increase by $\Delta P\left(V_{c}^{+}, V_{c}^{-}\right)$ in the area corresponding to the positive oriented domains. Similarly, when $V_{\mathrm{fe}}$ equals $V_{c}^{-}$this area decreases by $\Delta P\left(V_{c}^{+}, V_{c}^{-}\right)$. The polarization variation is then given by the product $2 P_{R} \cdot \Delta P\left(V_{c}^{+}, V_{c}^{-}\right)$, where $P_{R}$ is the remanent polarization.

As a final remark, the classical Preisach theory considers that the magnetic dipoles can have only two opposite orientations and all dipoles have the same contribution to the total magnetization. However, polycrystalline thin films have grains with more than one crystallographic orientation. Moreover, even for films with a predominant texture, e.g., $\langle 100\rangle$, there are domains with dissimilar oriented polarization, and therefore the ferroelectric domains have more than just two opposite orientations. The differently oriented domains do not have the same contribution to the total switched charge. In this experiment a highly $\langle 111\rangle$ oriented tetragonal phase $\mathrm{PbZr}_{0.3} \mathrm{Ti}_{0.7} \mathrm{O}_{3}$ was used. In this material the two different polarization directions (relative to the surface normal) equally contribute to the switching. For this particular case most of the domains have only two orientations with a good approximation.

\section{THE MODEL}

This article focuses on the hysteresis modeling, keeping the rest of the capacitor model as simple as possible, represented only by a linear capacitor and a resistor. As mentioned before, a good hysteresis model allows calculation of 


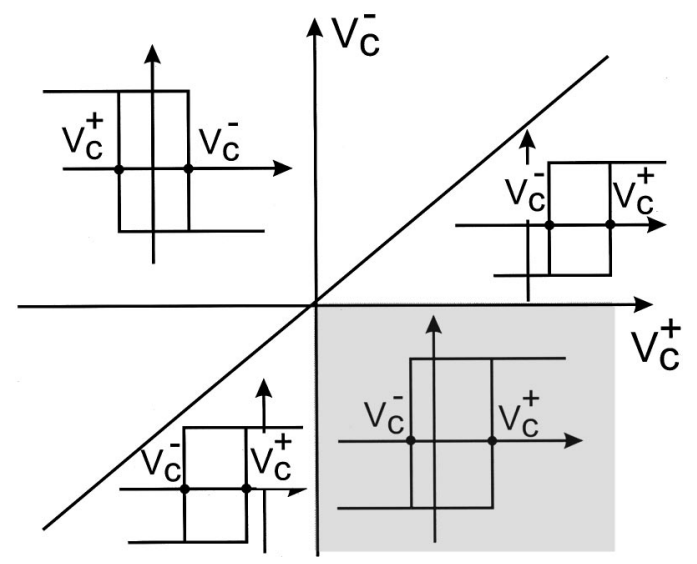

FIG. 3. A schematic representation of the hysteresis type corresponding to the different regions of the Preisach plane. The gray quadrant corresponds to the regular hysteresis loops. Outside it the distribution is assumed zero.

the polarization variations corresponding to arbitrary voltage changes. The Preisach model allows such calculations by providing a relation between the polarization and the voltage over the ferroelectric capacitor $V_{\text {fe }}$. As a potential disadvantage, it neglects any dynamic aspect of switching and this renders it inappropriate for simulations requiring a time accuracy better than the intrinsic switching time. However, the switching in thin films occurs very fast ${ }^{15}$ (hundreds of picoseconds) compared to the slopes of the signals in real circuits (a few nanoseconds) so that the detailed mechanism of switching can be neglected in a first approximation.

Although the Preisach distribution is defined over the whole Preisach plane, only a restricted area is involved in the polarization calculations. This area is found by analyzing the hysteresis type of the different regions of the Preisach plane. The lower-right quadrant of the plane corresponds to the normal situation of positive $V_{c}^{+}$and negative $V_{c}^{-}$. However, there is a half plane (see Fig. 3) for which $V_{c}^{+}<V_{c}^{-}$. These points correspond to domains that switch into the negative direction when $V_{\mathrm{fe}}$ is smaller than $V_{c}^{+}$. Although there might be reasons why this kind of domains could exist, our model does not take them into account because they cannot have a large influence, as proved by the experimental hysteresis loops, which always shows $V_{c}^{+}>V_{c}^{-}$. The last two regions correspond to both coercitive voltages positively, or both negatively. These cases correspond to the experimental cases of strongly imprinted hysteresis loops, which have only one stable state at zero voltage. For nonimprinted capacitors there will be only very few domains corresponding to these situations. Consequently, the Preisach distribution is assumed zero anywhere outside the lower-right quadrant and for continuity reasons, along the axes.

Furthermore, not all the domains from the lower right quadrant will be switched by $V_{\text {fe }}$. First, one should notice that a positive increasing voltage applied over the ferroelectric capacitor corresponds in the Preisach plane to a line moving parallel with the negative voltage axis. ${ }^{11}$ Similarly a negative decreasing voltage will correspond to a line moving downward parallel with the positive axis. As will be discussed further, it is assumed that positive decreasing or negative increasing voltages do not produce any switching. If 3.3

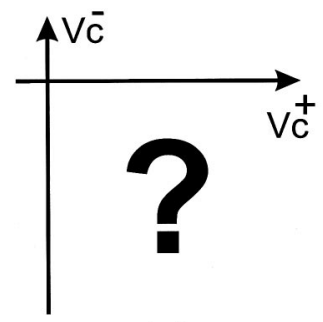

(a)

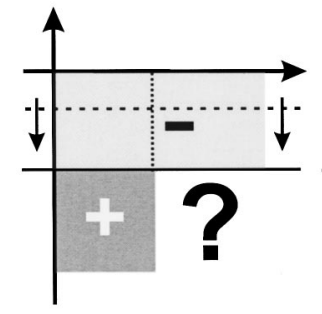

(c)

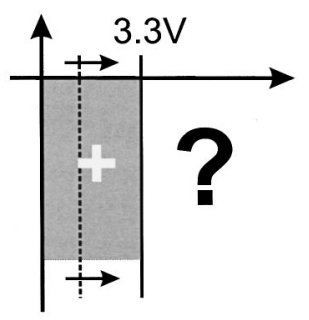

(b)

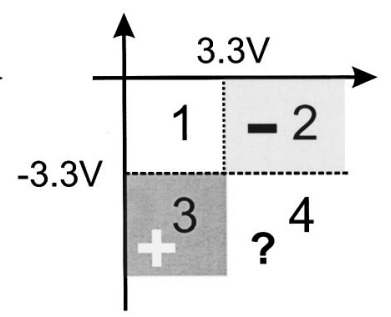

(d)
FIG. 4. Before applying any voltage: (a) the distribution corresponds to domains with different orientations. After the voltage rises to $3.3 \mathrm{~V}$ (b) the region behind this line will correspond to positive oriented domains. Similarly for $-3.3 \mathrm{~V}$ (c). If these are the maximum voltages, the measured signal will be determined only by domains corresponding to zone 1 (d).

$\mathrm{V}$ is applied the whole region between the negative axis and the $3.3 \mathrm{~V}$ line will correspond to positive oriented domains [see Fig. 4(b)] and similarly when $-3.3 \mathrm{~V}$ is reached [see Fig. 4(c)]. Assuming that these are the maximum voltages that will be used, zones 2 and 3 will remain negative, respectively, positive and the domains of zone 4 will keep their initial orientations for any further voltage [see Fig. 4(d)]. Only the domains inside zone 1 will actively participate in the measured signal.

The polarization can be calculated from the Preisach distribution, implementing a memory mechanism that uses, and thus has to memorize, only the voltage turning points and the corresponding polarization values. The detailed voltage shape between two turning points is not relevant for this model. After the previous sequence 3.3 and $-3.3 \mathrm{~V}$ are the first two turning points to be remembered as $V_{\mathrm{tp}}^{1+}$ and $V_{\mathrm{tp}}^{2-}$, respectively $P\left(V_{\mathrm{tp}}^{1+}\right)$ and $P\left(V_{\mathrm{tp}}^{2-}\right)$ (see Fig. 5). As the voltage rises the area occupied by the positive domains increases, leading to a charge flow through the circuit, the switching current. The polarization variations can be calculated from the Preisach distribution, by integrating over the area delimited by the two axes $V_{\text {fe }}$ and the previous turning point $V_{\mathrm{tp}}^{2-}$ in this case [see Fig. 5(a)]. ${ }^{10,11}$ It is obvious that the polarization variation rate is fully determined by the voltage, where the switching is considered instantaneous for the reasons mentioned before.

To simplify the implementation of the model we can make the bidimensional integral of the Preisach distribution, over the area limited by the axes and the maximum voltages, 3.3 and $-3.3 \mathrm{~V}$. In this case, the polarization variation is directly given by the value corresponding to the $\left(V_{\mathrm{fe}}, V_{\mathrm{tp}}^{2-}\right)$ point, $\Delta P\left(V_{\mathrm{fe}}, V_{\mathrm{tp}}^{2-}\right)$ (see Fig. 6). The polarization is obtained as 

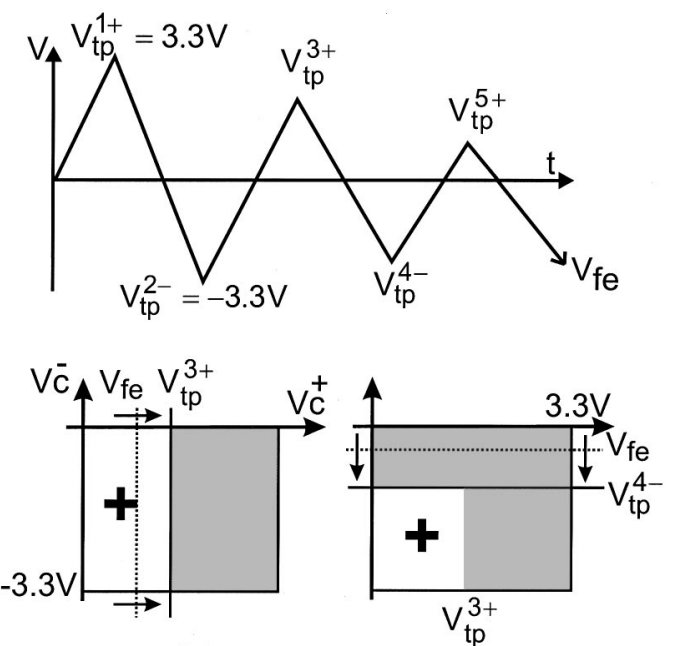

(a)

(b)

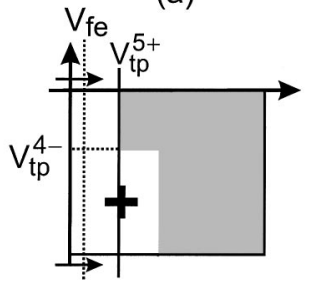

(c)

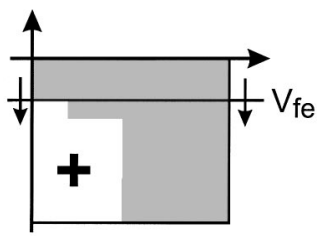

(d)
FIG. 5. The Preisach model allows the calculation of the polarization changes for an arbitrary input voltage, $V_{\mathrm{fe}}$. The voltage turning points are memorized and used for calculations. The polarization is given by the bidimensional integral over the area limited by the axes $V_{\mathrm{fe}}$ and the last turning point.

$$
P\left(V_{\mathrm{fe}}\right)=P\left(V_{\mathrm{tp}}^{2-}\right)+\Delta P\left(V_{\mathrm{fe}}, V_{\mathrm{tp}}^{2-}\right) .
$$

When the voltage starts decreasing, a new turning point is memorized $V_{\mathrm{tp}}^{3+}$. The model assumes no back switching and therefore no charge flows until the voltage reaches $0 \mathrm{~V}$. As the voltage decreases to negative values, only the positive oriented domains can be switched back [see Fig. 5(b)]. The rest of the domains are already negatively oriented and do not switch. The polarization is given similarly by

$$
P\left(V_{\mathrm{fe}}\right)=P\left(V_{\mathrm{tp}}^{3+}\right)-\Delta P\left(V_{\mathrm{tp}}^{3+}, V_{\mathrm{fe}}\right) \text {. }
$$

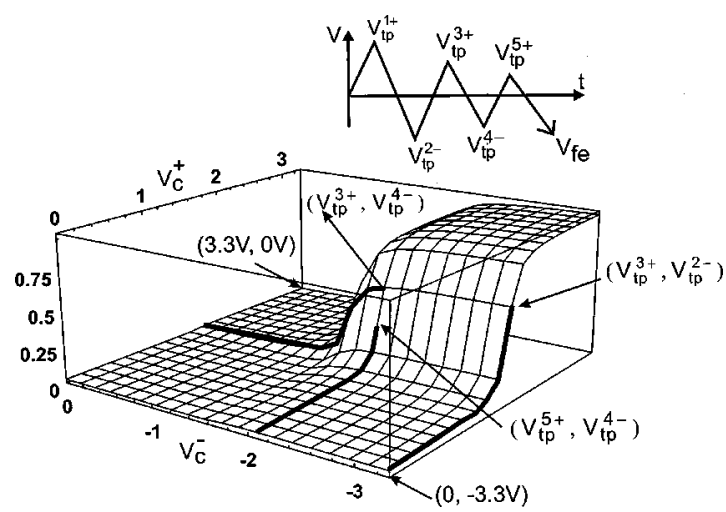

FIG. 6. To simplify the calculation of the polarization the Preisach distribution can be first integrated over the area of interest, usually set by the maximum voltages that will be applied. The polarization can then be directly calculated as the value corresponding to the voltage pair formed by $V_{\mathrm{fe}}$ and the last turning point.

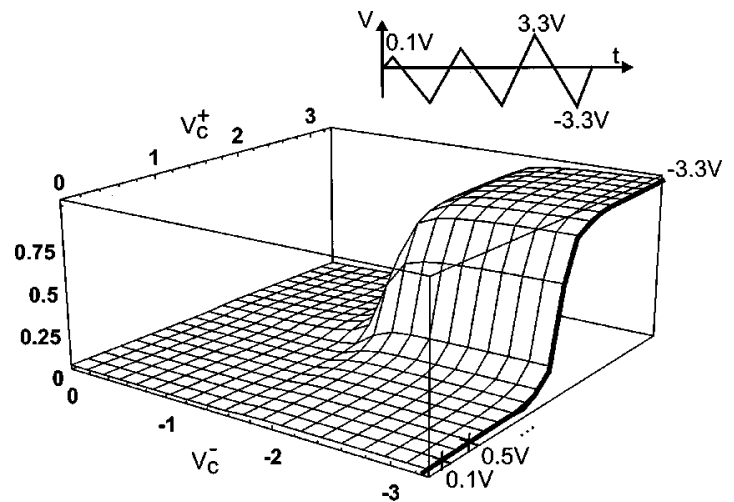

FIG. 7. The integrated Preisach distribution can be experimentally determined by applying a variable voltage (see inset) over the capacitor. For symmetric hysteresis loops the cross sections at -3.3 and $3.3 \mathrm{~V}$ are identical. In a first approximation the bidimensional distribution is obtained as the product between the two.

When the voltage starts to increase a second negative turning point is memorized; $V_{\mathrm{tp}}^{4-}$ [see Fig. 5(b)]. Because $V_{\mathrm{tp}}^{4-}$ is smaller than the previous negative turning point, there are more domains left oriented in the positive direction. If the field increases to positive values again the amount of switched charge will be smaller than in the first case, with fewer negative domains, generating a subloop included in the previous one. The polarization is calculated as

$$
P\left(V_{\text {fe }}\right)=P\left(V_{\text {tp }}^{4-}\right)+\Delta P\left(V_{\text {fe }}, V_{\text {tp }}^{4-}\right) .
$$

Providing that the voltage does not become larger than $V_{\mathrm{tp}}^{3+}$, $V_{\mathrm{tp}}^{5+}$ is added [see Fig. 5(c)].

Supposing the voltage decreases such that it becomes smaller than $V_{\mathrm{tp}}^{4-}$ the situation from before $V_{\mathrm{tp}}^{4-}$ is restored. The last two turning points $V_{\mathrm{tp}}^{5+}$ and $V_{\mathrm{tp}}^{4-}$ are no longer required for the polarization calculations [see Fig. 5(d)]. Their memory is lost. Eventually the voltage will reach $-3.3 \mathrm{~V}$ and the sample will be brought back to negative saturation. The whole memory is thus erased.

\section{EXPERIMENT}

This section discusses how the Preisach distribution can be determined experimentally. The sample used for measurements was a $\mathrm{PbZr}_{0.3} \mathrm{Ti}_{0.7} \mathrm{O}_{3}$ thin film, of $200 \mathrm{~nm}$ thickness, prepared as described in Ref. 16. The sample was first poled. It is known that the hysteresis loops before and after poling look very different, meaning changes in the distribution and rising questions about its stability. However, after poling, if no degradation phenomena such as imprint or fatigue occur, the measurements are very reproducible so it can be concluded that the distribution no longer changes. The poling voltage was a signal of $3.3 \mathrm{~V}$ amplitude because the model will be used to simulate a memory cell for $0.35 \mu \mathrm{m}$ complementary metal-oxide-semiconductor technology, with a maximum operating voltage of $3.3 \mathrm{~V}$. Poling at a higher voltage changes the domain wall configuration and consequently the distribution, leading to inaccurate simulations.

The next step is to apply a voltage oscillating between $-3.3 \mathrm{~V}$ and increasing positive values, starting from 0.1 to $3.3 \mathrm{~V}$ (see Fig. 7). The measurement was performed with a 


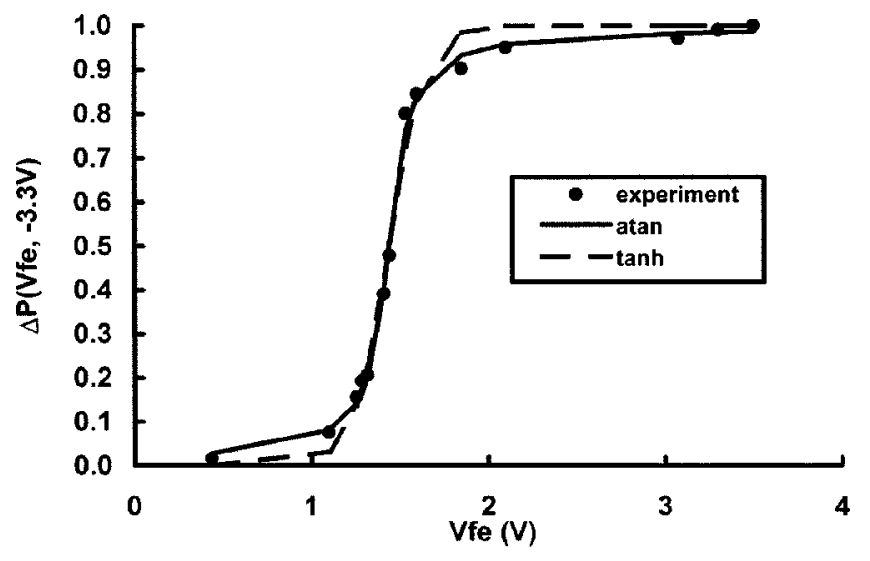

FIG. 8. The cross section of the Preisach distribution at $-3.3 \mathrm{~V}$ and the fittings, using atan and tanh. For this particular sample atan gives better fitting results.

modified Sawyer-Tower setup and thus the measured signal is the switched charge, that is the integral of the Preisach distribution. Let us assume that after the sample is poled into negative saturation. Then, the voltage was increased to $0.1 \mathrm{~V}$ and the corresponding switching charge is recorded $Q(0.1$, $-3.3 \mathrm{~V})$. This charge is obtained as the difference between the $0 \mathrm{~V}$ polarizations, measured when voltage rises from -3.3 to $0.1 \mathrm{~V}$, respectively, and decreases from 0.1 to -3.3 $\mathrm{V}$. In this way the reversible contributions (linear part) are eliminated. The charge is then normalized to the maximum switched charge $Q(3.3,-3.3 \mathrm{~V})$. In the integrated Preisach diagram this value corresponds to the point $(0.1,-3.3 \mathrm{~V})$. Next the memory is erased by a voltage going to $-3.3 \mathrm{~V}$. The next point is acquired by raising the voltage until $0.5 \mathrm{~V}$ this time. The switched charge is recorded $Q(0.5,-3.3 \mathrm{~V})$, and the value of the integrated Preisach distribution is calculated again by normalization. Similarly the whole cross section of the distribution integral at $-3.3 \mathrm{~V}$ is measured in this way (see Fig. 7). These data, normalized to the maximum value obtained for $(3.3,-3.3 \mathrm{~V})$, are shown in Fig. 8. By normalization, the same distribution can be used for samples with different values of $P_{R}$, assuming that it can be scaled. For symmetric hysteresis loops the same cross section is obtained at $3.3 \mathrm{~V}$.

To make the implementation of the model easier, the experimental data points are fitted with an analytic function. Because the theoretical determination of the Preisach distribution or its integral is beyond the scope of this article, the analytical function that is used for the fitting is not important. The results for tanh and atan functions are shown in Fig. 8. For this particular sample, atan shows a better fitting. In this case the only parameters are the coercitive voltage $V_{c}$ $=1.4 \mathrm{~V}$, and $a=11.3$ describing the steepness of the distribution. If we define $f\left(V_{\mathrm{fe}}, V_{c}\right)$ as

$$
f\left(V_{\mathrm{fe}}, V_{c}\right)=2 \times \operatorname{atan}\left(a \times\left(V_{\mathrm{fe}}-V_{c}\right)\right) / \pi,
$$

then the cross section of the distribution at $-3.3 \mathrm{~V}$ is given by $\left[1+f\left(V_{\mathrm{fe}}, V_{c}^{+}\right)\right] / 2$, and at 3.3 by $\left[1-f\left(V_{\mathrm{fe}}, V_{c}^{-}\right)\right] / 2$. In the so-called linear approximation, the distribution for intermediate voltages can be considered equal to the product of these functions

$$
\Delta P\left(V_{x}, V_{y}\right)=P_{R}\left[1+f\left(V_{x}, V_{c}^{+}\right)\right]\left[1-f\left(V_{y}, V_{c}^{-}\right)\right] / 2 .
$$

This gives a good description of the saturated hysteresis loops, but the agreement is not so good for the subloops, because in general the fitting parameters $a$ and $V_{c}$ are not voltage independent. In order to determine their dependence the whole bidimensional distribution has to be measured. This measurement can be done using the above described method, and these results will be presented into a future article. However, this method allows a fast implementation of the model with minimum measuring effort and good simulation results.

\section{THE SIMULATION RESULTS}

To verify the model the experimental and the simulated hysteresis loops have to be compared. The model has been implemented using the Spectre Hardware Description Language ${ }^{1},{ }^{14}$ which allows custom models to be written for the Spectre $\circledR$ simulator. This circuit simulator is integrated in the Cadence ${ }^{\circledR}{ }^{17}$ environment, which is one of the standard design environments for the semiconductor industry. The simulated circuit reproduced the Sawyer-Tower circuit used for measurements. The ferroelectric capacitor was modeled using the Preisach model, in parallel with a linear capacitor and a resistor. The voltage turning points and the corresponding polarization values are memorized in an array and used to calculate the polarization as a function of voltage. The measured Preisach distribution is described analytically by the product of the two functions normalized to unity, as explained in the Experiment section. The polarization can be calculated using the memory mechanism described in the model part and the following formulas:

$$
\begin{aligned}
P\left(V_{\mathrm{fe}}\right)= & P_{\mathrm{tp}}^{n}+0.5 P_{R} \cdot\left[1+f\left(V_{\mathrm{fe}}, V_{c}^{+}\right)\right] \\
& \cdot\left[1-f\left(V_{\mathrm{tp}}^{n}, V_{c}^{-}\right)\right] \text {for positive rising } V_{\mathrm{fe}}, \\
P\left(V_{\mathrm{fe}}\right)= & P_{\mathrm{tp}}^{n}-0.5 P_{R} \cdot\left[1+f\left(V_{\mathrm{tp}}^{n}, V_{c}^{+}\right)\right] \\
& \cdot\left[1-f\left(V_{\mathrm{fe}}, V_{c}^{-}\right)\right] \text {for negative decreasing } V_{\mathrm{fe}},
\end{aligned}
$$

where $V_{\mathrm{tp}}^{n}$ and $P_{\mathrm{tp}}^{n}$ are the voltage and polarization at the last turning point, $V_{\mathrm{fe}}$ is the voltage over the ferroelectric capacitor and can have any arbitrary variation, and $P_{R}$ is the remanent polarization. One can notice that the parenthesis containing $V_{\text {tp }}^{n}$ plays the role of a scaling factor and it represents the amount of domains that can be switched. The switching current is given by

$$
I_{\mathrm{sw}}=S \cdot \frac{\partial P\left(V_{\mathrm{fe}}\right)}{\partial V_{\mathrm{fe}}} \cdot \frac{\partial V_{\mathrm{fe}}}{\partial t},
$$

where $S$ is the area of the capacitor.

The simulated results reproduce very well the measurement for saturated hysteresis loops, but show some differences for the partial loops (see Fig. 9). As mentioned before these differences are mainly due to the linear approximation made for the Preisach distribution. More detailed measure- 


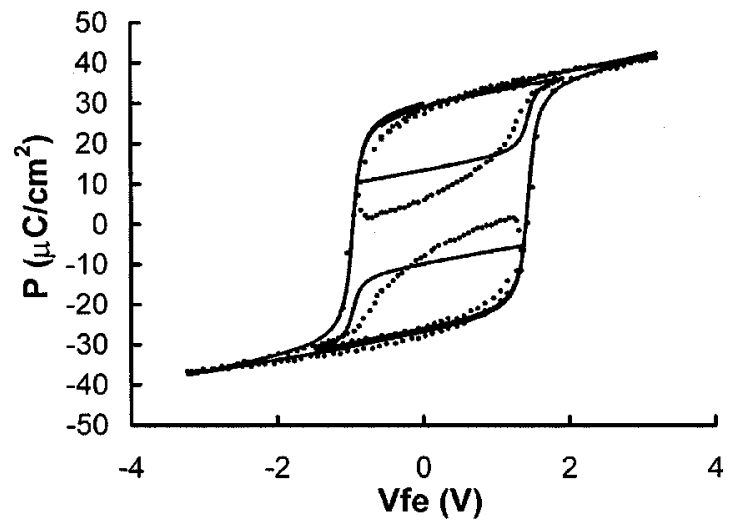

FIG. 9. Comparison between experimental and simulated hysteresis loop. There is very good agreement for saturated loops and the differences observed for the subloops are due to the linear approximation used for the Preisach distribution.

ments will be carried out in the future, in order to experimentally obtain the full bidimensional distribution which will improve the simulation results.

\section{CONCLUSIONS}

Before applying the Preisach theory to the ferroelectric materials its suppositions need to be verified for the sample under investigation. The Preisach distribution is related to the polarization variations corresponding to a coercitive voltage pair. The polarization is given by the value of the integrated Preisach distribution at the point corresponding to the voltage over the ferroelectric and the previous turning point. The memory mechanism is implemented by storing the voltage turning points and the corresponding polarizations in an array and deleting them in pairs when the voltage becomes higher than the last positive turning point, or lower than the last negative turning point. The Preisach distribution was experimentally determined in a first order approximation, which has as a main limitation a limited accuracy in the subloops simulations. The implementation in the Spectre ${ }^{\circledR}$ circuit simulator of the model gives good simulation results and can be used for ferroelectric memory design.

${ }^{1}$ E. Fatuzzo and W. J. Merz, Phys. Rev. 116, 61 (1959).

${ }^{2}$ Y. Ishibashi and Y. Takagi, J. Phys. Soc. Jpn. 31, 506 (1971).

${ }^{3}$ A. K. Kulkarni, G. A. Rohrer, S. Narayan, and L. D. McMillan, Ferroelectrics 116, 95 (1991)

${ }^{4}$ K. Dimmler, M. Parris, D. Butler, S. Eaton, B. Pouligny, J. F. Scott, and Y. Ishibashi, J. Appl. Phys. 61, 5467 (1987).

${ }^{5}$ J. F. Scott, L. Kammerdiner, M. Parris, S. Traynor, V. Ottenbacher, A. Shawabkeh, and W. F. Oliver, J. Appl. Phys. 64, 787 (1988).

${ }^{6}$ M. E. Drougard, J. Appl. Phys. 31, 352 (1960).

${ }^{7}$ C. J. Dias and D. K. Das Gupta, J. Appl. Phys. 74, 6317 (1993).

${ }^{8}$ A. T. Bartic, D. J. Wouters, and H. E. Maes, Integr. Ferroelectr. 22, 171 (1998).

${ }^{9}$ S. L. Miller, R. D. Nasby, J. R. Schwank, M. S. Rodgers, and P. V. Dressendorfer, J. Appl. Phys. 68, 6463 (1990).

${ }^{10} \mathrm{~J}$. T. Evans and J. A. Bullington, IEEE 7th International Symposium on Applications of Ferroelectrics, New York, 1991, p. 692.

${ }^{11}$ I. D. Mayergoyz, Mathematical Models of Hysteresis (Springer, New York, 1991).

${ }^{12}$ B. Jiang, P. Zurcher, R. E. Jones, S. J. Gillespie, and J. C. Lee, Symposium on VLSI Technology, 1997, p. 141.

${ }^{13}$ H. Goebel, M. Ullmann, G. Schindler, and M. Kastner, International Conference on Solid State Devices and Materials, 1999, p. 386.

${ }^{14}$ SpectreHDL Reference, Cadence Design Systems, 1997.

${ }^{15}$ P. K. Larsen, G. L. M. Kampschoer, M. B. van der Mark, and M. Klee, Proceedings of the Eighth IEEE International Symposium on Applications of Ferroelectrics, 1992, p. 217.

${ }^{16}$ D. J. Wouters, G. Willems, E. G. Lee, and H. Maes, Integr. Ferroelectr. 15, 79 (1996).

${ }^{17}$ OpenBook version 4, Cadence Design Systems (1998). 